\title{
Assessment of pupillary light reflex latency and darkness adapted pupil size in control subjects and in diabetic patients with and without cardiovascular autonomic neuropathy
}

\author{
P Lanting, J E Bos, J Aartsen, L Schuman, J Reichert-Thoen, J J Heimans
}

\begin{abstract}
Increased pupillary light reflex latencies were found more often than a reduced darkness pupil size in diabetic patients with and without abnormal cardiovascular reflexes. This finding suggests that parasympathetic pupillary dysfunction precedes sympathetic pupillary denervation in diabetic autonomic neuropathy.
\end{abstract}

Measurement of pupillary function may provide information on the function of the autonomic nervous system. Darkness adapted pupil size may be reduced in diabetic patients indicating a failure of sympathetically mediated dilatation. ${ }^{1}$ The latency of the pupillary light response is increased $^{23}$ in diabetic patients, indicating reduced parasympathetic function. ${ }^{3}$ We have compared sympathetic and parasympathetic nervous system input to the iris with cardiovascular reflex activity in diabetic patients. Sympathetic and parasympathetic pupillary innervation were assessed by measuring darkness pupil size and pupillary light reflex latency respectively. In determining pupillary light reflex latency we used the IRIS infrared light reflex technique. ${ }^{4}$ This method combines an "open loop" stimulatory technique, in which pupil size does not influence the light input on the retina, with the possibility of stimulating a large symmetrical retinal area of about $30^{\circ}$. In this way, the pupillary light reflex is less influenced by local retinal abnormalities, which may be present particularly in diabetic patients, due to retinopathy.

It was our aim to investigate to what extent determination of pupillary light reflex latency and darkness adapted pupil size contribute to the diagnosis of autonomic neuropathy in diabetes mellitus.

Department of Ophthalmology J Reichert-Thoen

Correspondence to: $P$ Lanting, Department of Neurology, Free University Neurology, Free University 1081 HV Amsterdam, The Netherlands.

Received 17 May 1989 and in final revised form 7 March 1990.

Accepted 15 March 1990
Pupillary light reflex latency was investigated in fifty six non-diabetic subjects, 28 women and 28 men, aged from 12 to 75 years (mean age 39.3 years). In 49 , darkness adapted pupil size was also measured. All non-diabetic persons were healthy and were not receiving any medication.

Thirty diabetic patients participated in the study. Fifteen patients had normal cardiovascular reflexes [group 1, age: mean (SD) 44.7 (14.5) years; duration of diabetes: mean (SD) 15.8 (15.9) years]. In fifteen patients cardiovascular reflex activity was abnormal [group 2, age: mean (SD) 50.6 (12.7) years; duration of diabetes: mean (SD) $17.5(12.6)$ years]. The following cardiovascular reflex tests were assessed: heart rate response to standing $\left(\mathrm{HR}_{30-15}\right.$ ratio) and heart rate variation with deep breathing (I-E value). Both tests have been described previously. ${ }^{5}$ The reference values were obtained from 65 healthy persons. Values above the lower onesided $95 \%$ confidence limits were considered to be in the normal range. $\mathrm{HR}_{30-15}$ ratio ranged from 1.02 to 1.65 , mean (SD) $1.25(0.23)$, and from 0.90 to 1.01 , mean (SD) $0.97(0.03)$ in groups 1 and 2, respectively. I-E value ranged from 11 to 31 beats per minute, mean (SD) $18.4(6 \cdot 1) \mathrm{bpm}$ and from two to $10 \mathrm{bpm}$, mean (SD) $6(2 \cdot 2) \mathrm{bpm}$ in group 1 and 2, respectively.

Patients were excluded from the study if they had neuropathy that was not of diabetic origin. None of the patients was taking drugs known to interfere with autonomic function. Ophthalmological examination revealed small retinal haemorrhages and/or exudates in four patients in group 2. The presence of retinopathy was assessed by dilated fundoscopy and photographs of both fundi of standard field 1 and 2 as described by the Diabetic Retinopathy Study Research group. ${ }^{6}$ Visual acuity was at least $6 / 9$.

The pupillary light reflex latency was measured with the IRIS infrared light reflex technique. ${ }^{4}$ The retina was stimulated with a block-shaped stimulus with a fixed background and step intensity, corresponding to a retinal illuminance of respectively $1.2 \mathrm{Log}$ Troland and 3.7 Log Troland. The duration of the stimulus was $1 \cdot 2 \mathrm{~s}$. The time interval between two consecutive stimuli was $5 \mathrm{~s}$. The latency of the pupillary constriction was measured from at least six artefact-free 
Figure 1 Pupillary light reflex latency in 15 and 15 patients without

(O) abnormal

cardiovascular reflex tests. The dotted lines show the $95 \%$ confidence limits on the regression line calculated from 56 healthy persons. diabetic patients with $(\bullet$

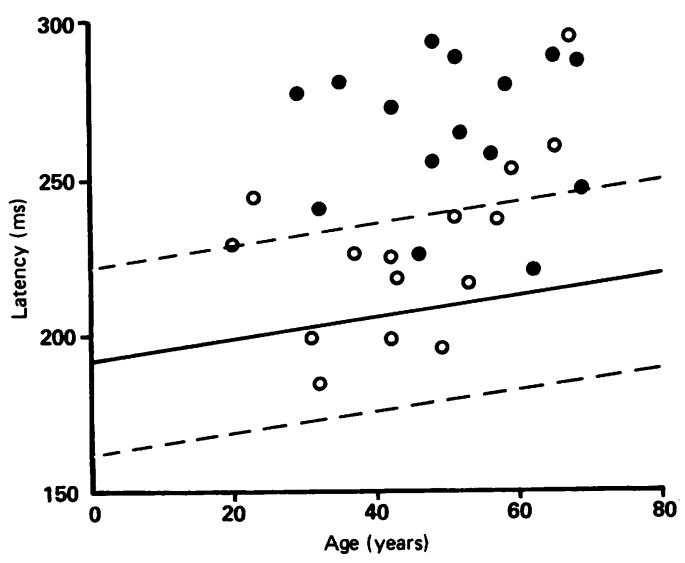

responses of both eyes. All pupillary measurements were performed in darkness to which the subjects had adapted for two minutes.

Darkness adapted pupil size was measured using a slitlamp camera. After focussing the pupil and iris with a three stage light intensifier system (infrared light was used as a light source), a photograph was taken using electronic flashlight. The pupil diameter percentage (PD $\%$, horizontal pupil diameter as a percentage of iris diameter) was calculated from the photographs, which magnified pupil and iris $2 \cdot 5$-fold. Experiments in 15 control subjects had established that there was no difference in $\mathrm{PD} \%$ after two and 20 minutes of darkness adaptation. In eight diabetic patients and 10 control persons darkness pupil size and pupillary latency were measured with an interval of one week to evaluate intraindividual variability.

Blood glucose levels were measured at the beginning of the pupillometry sessions and none of the patients had hypoglycaemia.

\section{Statistical analysis}

Normal values, which were defined as values within the $95 \%$ confidence limits, were determined using a linear regression equation. Differences between both patient groups concerning latency of pupillary constriction, $\mathrm{PD} \%$ and age were determined by multiple regression analysis. The coefficient of variation for day-to-day variation of $\mathrm{PD} \%$ and latency of pupillary constriction in the same subject was computed.
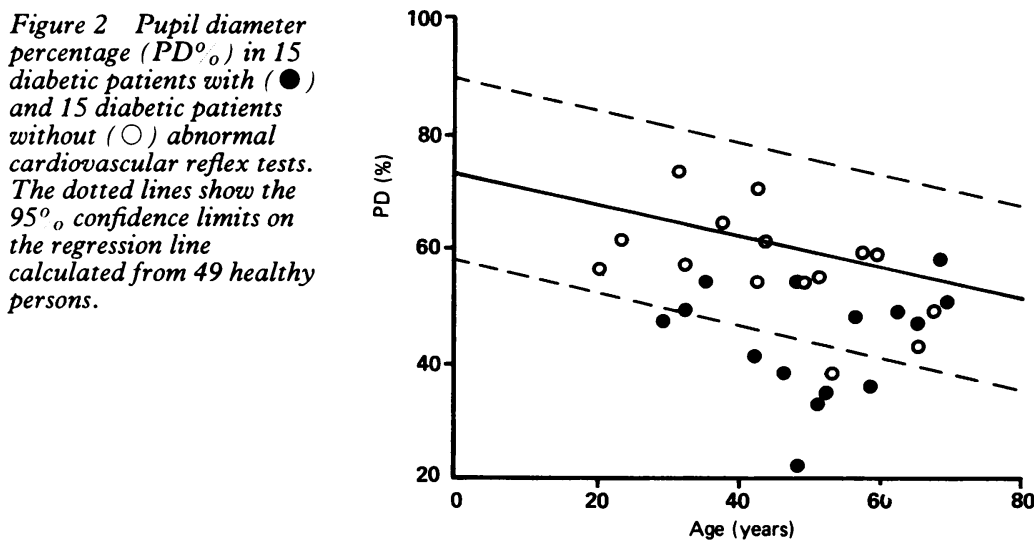

\section{Results}

Latencies of pupillary constriction in the non diabetic group ranged from 179 to $246 \mathrm{~ms}$, mean (SD) $205.9(15.4) \mathrm{ms}$. There was a positive relationship between pupillary latency and ageing $(\mathrm{r}=0.40, \mathrm{p}<0.01) . \mathrm{PD} \%$ in the non-diabetic group ranged from 43 to $84 \%$, mean (SD) $63 \%(8 \cdot 3)$, and showed a significant decrease with ageing $(\mathrm{r}=0.49, \mathrm{p}<0.01)$.

The coefficient of variation of $\mathrm{PD} \%$ was, mean (SD) $3.2(0.9 \%)$ in the control subjects and $4(1 \cdot 1 \%)$ in the diabetic patients. The coefficient of variation of latency of pupillary constriction, mean (SD) was $3.8(2.4 \%)$ in the control subjects and $5.6(4 \%)$ in the diabetic patients.

Thirteen patients in group 2, and four patients in group 1 had a prolonged pupillary latency period (fig 1). PD\% was abnormally small in seven patients in group 2 and in one patient in group 1 (fig 2). Latencies of pupillary constriction in group 2 were significantly prolonged compared with those in group 1 , mean (SD): $266.7 \mathrm{~ms} \mathrm{(23.9)} \mathrm{and} 228.8 \mathrm{~ms}(28.9)$ respectively, $p<0.001)$. PD \% was significantly smaller in group 2 compared with group 1 , mean (SD): $45.1 \%(9.7)$ and $57.9 \%(9.4)$ respectively, $p<0.01$ ). Latencies of pupillary constriction and $\mathrm{PD} \%$ in the diabetic patients were inversely correlated $(r=-0.38$, $\mathrm{p}<0.05$ ).

If the results of both cardiovascular reflex tests are considered to be an indication of whether or not autonomic neuropathy is present, the sensitivity of the pupillary latency as a diagnostic test is $\mathbf{8 6 . 7 \%}$ and the specificity $73.3 \%$, whereas the sensitivity of $\mathrm{PD} \%$ is $46 \cdot 7 \%$ and the specificity $93 \cdot 3 \%$.

\section{Discussion}

The age dependence of darkness adapted pupil size and pupillary light reflex latency that we found, has been observed by others and indicates that both sympathetic and parasympathetic input on the iris muscles decrease with ageing. ${ }^{7}$

In both groups of patients, with normal or abnormal results for the cardiovascular reflex tests, pupillary light reflex latency was more often abnormal than darkness adapted pupil size. Increased latencies of pupillary constriction may be due to increased sympathetic tone or decreased parasympathetic function. ${ }^{3}$ As shown in fig 2 there were no patients with increased sympathetic tone. This means that the increased pupillary light reflex latencies in the patients are a result of decreased parasympathetic function.

Because structural changes have been observed in the iris muscles of diabetic patients, ${ }^{8}$ it would not be surprising if these changes were found to influence pupil size and pupillary light reflex latency. However, because the diabetic pupil shows enhanced responses to cholinomimetic ${ }^{9}$ and sympathicomimetic $^{10}$ agents, it is not likely that mechanical factors restrict pupil motility in diabetic patients.

It cannot be ruled out that the presence of 
background retinopathy in four patients, all of whom had abnormal cardiovascular reflex tests, could have contributed to prolongation of latency of pupillary constriction. However, the stimulation technique used minimised the influence of retinal pathology.

Our findings suggest that parasympathetic dysfunction occurs before sympathetic dysfunction in the pupillary reflex arc of diabetic patients. This finding agrees with the sequence in which cardiovascular reflex abnormalities occur in diabetic patients. In contrast to our findings and to those of others, ${ }^{23}$ Hreidarsson could not demonstrate increased pupillary light reflex latencies in diabetic patients. ${ }^{11}$ This could be due to the fact that a television pupillometer was used which had a lower time resolution than our recording technique. ${ }^{4}$ Moreover, only a small retinal area was stimulated, which may have contributed to the wide range in the normal data.

In conclusion, our study suggests that, compared with cardiovascular reflex tests, determination of pupillary latency with the IRIS registration technique is a sensitive test of autonomic function. Pupillary latency may be prolonged in diabetic patients with normal cardiovascular reflex activity. This implies that assessment of pupillary innervation can provide additional information on the function of the autonomic nervous system.

We thank Drs J J P Nauta for his statistical advice and Mrs A M M Op't Eijnde-van Dolen for preparing the manuscript.

1 Smith SA, Dewhirst R. A simple diagnostic test for pupillary abnormality in diabetic autonomic neuropathy. Diabet abnormality in diabet

2 Friedman SA, Feinberg R, Podolak E, Bedell RHS. Pupillary abnormalities in diabetic neuropathy. A preliminary study. Ann Intern Med 1967;67:977-83.

3 Pfeifer MA, Cook D, Brodsky J, et al. Quantitative evaluation of sympathetic and parasympathetic control of iris function. Diabetes Care 1982;5:518-28.

4 Reulen JPH, Marcus JT, van Gilst MJ, et al. Stimulation and recording of dynamic pupillary reflex: The IRIS and recording of dynamic pupillary refex: The IR

5 Ewing DJ, Clarke BF. Diagnosis and management of diabetic autonomic neuropathy. $\mathrm{Br}$ Med $J$ 1982;285: 916-18.

6 Diabetic Retinopathy Study Research group. Report 7. A modification of the Airly House classification of diabetic modification of the Airly House classification of diabetic
retinopathy. Invest Ophthalmol Vis Sci 1981;21:210-26.

retinopathy. Invest Ophthalmol Vis Sci 1981;21:210-26.
7 Pfeifer MA, Weinberg CR, Cook D, Best JD, Reenan A, Halter JB. Differential changes of autonomic nervous system function with age in man. Am J Med 1983;75: 249-258.

8 Fujii T, Ishikawa S, Uga S. Ultrastructure of Iris Muscles in Diabetes Mellitus. Ophthalmologica, (Basel) 1977;174: 228-39.

9 Sigsbee B, Torkelson R, Kadis G, Wright JW, Reeves AG. Parasympathetic denervation of the iris in diabetes
mellitus. J Neurol Neurosurg Psychiatry 1974;37:1031-5.

10 Smith SA, Smith SE. Evidence for a neuropathic aetiology in the small pupil of diabetes mellitus. Br J Ophthalmol in the small pupil

11 Hreidarsson AB, Gundersen HJG. The pupillary response to light in Type 1 (insulin-dependent) diabetes. Diabetologia 1985;28:815-21. 